\title{
An atomistic view on liquid phase adsorption
}

\author{
Hamzeh Kraus • Niels Hansen
}

Received: date / Accepted: date

\begin{abstract}
The effect of immobilized $\beta$-cyclodextrin (bCD) molecules inside a mesoporous silica support on the uptake of benzene and $p$-nitrophenol from aqueous solution was investigated using all-atom molecular dynamics (MD) simulations. The calculated adsorption isotherms are discussed with respect to the free energies of binding for a 1:1 complex of bCD and the aromatic guest molecule. The adsorption capacity of the bCDcontaining material significantly exceeds the amount corresponding to a 1:1 binding scenario, in agreement with experimental observations for benzene adsorption. The demonstrated feasibility of classical all-atom MD simulations to calculate liquid phase adsorption isotherms paves the way to a molecular interpretation of experimental data that are too complex to be described by empirical models.
\end{abstract}

Keywords Silica pore - Cyclodextrin · Adsorption · Molecular Dynamics Simulation

\section{Introduction}

Cyclodextrins (CDs) are cyclic oligosaccharides with conical shape built of (1-4)-linked $\alpha$-D-glucopyranoside units. Their hydrophilic outer surface renders them soluble in water, while the hydrophobic interior can accommodate hydrophobic compounds. Therefore, they are employed in the removal of pollutants from wastewater [1], as chiral selectors [2] or in (asymmetric) supramolecular catalysis [3-9] among many other fields of applications $[10,11]$. By tuning the size and shape of the hydrophobic cavity through derivatization of the native

Institute of Thermodynamics and Thermal Process Engineering, University of Stuttgart, D-70569 Stuttgart, Germany. Tel: +49 711 685-66112; E-mail: hansen@itt.uni-stuttgart.de cyclodextrins the selectivity towards the target compounds can be increased [12]. In addition, the ease of functionalization of their scaffold allows to introduce additional catalytic groups or binding sites in specific positions [13]. Together with the solvent used and a possible immobilization of the cyclodextrins on a solid support a complex multidimensional design problem emerges. Several approaches can be envisioned to produce CD-functionalized materials. The first involves crosslinking of CDs into polymers using C-OH linkers [14]. A second approach utilizes the coating or grafting of CD moieties onto a stationary phase such as organic polymers or silica gel [15]. A third type of material is based on a mesoporous silica support. Mesoporous silicas with chemically attached macrocyclic moieties were successfully prepared by sol-gel condensation of tetraethyl orthosilicate and $\beta$-cyclodextrin-silane in the presence of a structure directing agent [16-19], resulting in silica-based materials that possess a uniform framework mesoporosity with defined nanoscaled cavities. The ability of removing aromatic compounds from an aqueous phase was investigated, and it was concluded that the synthesized materials is promising for this purpose $[16,17,19]$.

Molecular modelling approaches are particularly feasible to study the delicate balance between the various intermolecular forces determining macroscopic behavior and allow a fundamental understanding at the molecular level. Gas adsorption in microporous materials such as zeolites has been studied for more than 45 years in particular using classical molecular simulations [20-22]. The rich diffusion behaviour in nanoporous solid materials has recently been reviewed [23]. The investigation of liquid phase systems was largely driven by studies related to liquid chromatography, i.e., partitioning behavior of (multicomponent) mixtures at a solid surface 
functionalized for example with alkyl chains [24-27]. Other studies used coarse-grained molecular dynamics (MD) simulations to investigate protein adsorption on solid supports $[28,29]$ including the calculation of adsorption isotherms. An atomistic simulation study of enantiomeric separation of (R)- and (S)-ibuprofen in methanol solvent by means of immobilized cyclodextrins in a slit shaped model pore was reported recently [30]. Therefore, molecular simulation has evolved toward a promising tool to study liquid phase separation processes that are too complex to be described by phenomenological models [31].

The aim of the present work is to investigate the feasibility of all-atom classical MD simulations to reconcile liquid phase adsorption experiments with theoretical predictions. For this purpose adsorption of benzene and $p$-nitrophenol from aqueous solutions onto cyclodextrinfunctionalized mesoporous silica support is modelled and analyzed. Binding free energy calculations in bulk solvent are related to the Henry regime of the adsorption isotherm on the functionalized material. The interpretation of experimentally observed adsorption isotherms is discussed in view of the underlying molecular level picture.

\section{Methodology}

2.1 Calculation of binding free enthalpies and rate constants in bulk solution

Two approaches were employed to calculate binding free enthalpies, an unbiased (counting) one and a biased (double decoupling) one.

In the unbiased approach, referred to as direct counting (DC), the occurrences of bound, $N_{\mathrm{b}}$, and unbound, $N_{\mathrm{u}}$, instances during long $(t \geq 10 \mu \mathrm{s})$ standard MD simulations of one host-guest pair solvated in a box of water are counted. The binding free enthalpy is then obtained from $[32]$

$\Delta G^{\mathrm{DC}}=-R T \ln \frac{N_{\mathrm{b}}}{N_{\mathrm{u}}}-R T \ln \frac{V_{\mathrm{box}}}{V^{0}}$

with standard state volume $V^{0}=1.661 \mathrm{~nm}^{3}$ and average volume of the simulation box $V_{\mathrm{box}}$. In addition, average bound $\left\langle t_{b}\right\rangle$ and unbound $\left\langle t_{u}\right\rangle$ residence times can be calculated, yielding association $k_{\text {on }}$ and dissociation $k_{\text {off }}$ rates $[32]$

$k_{\mathrm{on}}=\frac{1}{\left\langle t_{\mathrm{u}}\right\rangle C_{\mathrm{g}}}, \quad k_{\mathrm{off}}=\frac{1}{\left\langle t_{\mathrm{b}}\right\rangle}$

with guest molecule concentration $C_{\mathrm{g}}$. The binding free enthalpy can then be determined using these rate con- stants $(\mathrm{RC})$

$$
\begin{aligned}
\Delta G^{\mathrm{RC}} & =-R T \ln \frac{k_{\mathrm{on}} C^{0}}{k_{\mathrm{off}}} \\
& =-R T \ln \frac{\left\langle t_{\mathrm{b}}\right\rangle}{\left\langle t_{\mathrm{u}}\right\rangle}-R T \ln \frac{V_{\mathrm{box}}}{V^{0}}
\end{aligned}
$$

with standard state concentration $C^{0}=1 \mathrm{moll}^{-1}$.

In order to identify bound and unbound instances, the host and guest molecule structures need to be geometrically reduced to comparable reference points. Using the different glucopyranose units, the conical shape of cyclodextrin was first reduced to three main circles, one running through the oxygen atoms of the primary hydroxyl groups, one central circle passing through the central carbon atoms and one circle connecting the oxygen atoms of the secondary hydroxyl groups. This system was then further reduced to a three point system based on the centers of mass of the different circles. Similarly, the two guest molecules were also reduced to a three point system as illustrated in figure 1 .

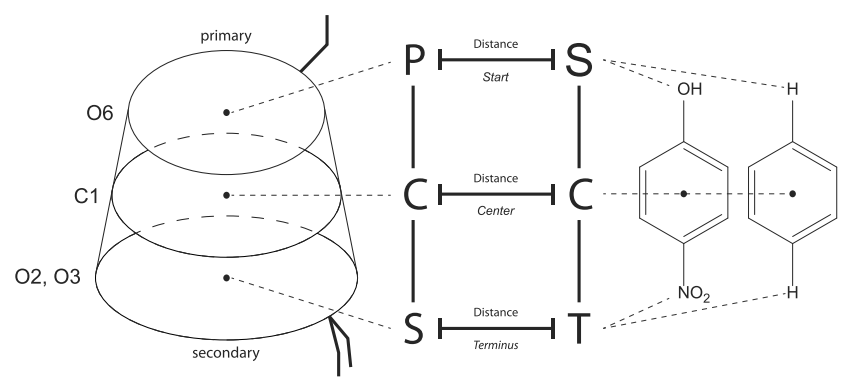

Fig. 1 Geometrical representation of the cyclodextrin and guest molecule structures by a three point system.

To determine whether a configuration is in a bound state, a spatial cut-off was defined for the central distance within which the guest molecule is assumed to be bound to the host molecule. Monitoring the angle of the two three point systems provides the orientation in which the guest molecule is bound to the host. Observing the bound and unbound state over time results into bound and unbound instances of different duration. Averaging over these instances yields time averages $\left\langle t_{\mathrm{b}}\right\rangle$ and $\left\langle t_{\mathrm{u}}\right\rangle$. Optionally, a temporal cut-off can be introduced that removes bound and unbound instances with a smaller residence time from the averaging. The impact of the temporal cut-off is shown in the supplementary material in table $\mathrm{S} 1$ and discussed below.

The second method used for calculating the binding free enthalpy was double decoupling (DD) [33]. As illustrated in figure 2 the process is divided into two parts. First, the free enthalpy difference $\Delta G_{\mathrm{u}}^{\mathrm{M} \rightarrow \mathrm{M}^{\prime}}$ is calculated, resulting from decoupling the unbound state $\mathrm{u}$, 
i.e., turning off the intermolecular interactions with the environment $\left(\mathrm{M} \rightarrow \mathrm{M}^{\prime}\right)$ of the guest molecule in a box of water while preserving intramolecular interactions. $\Delta G_{\mathrm{u}}^{\mathrm{M} \rightarrow \mathrm{M}^{\prime}}$ is equal to the negative hydration free enthalpy $-\Delta G_{\text {hyd }}^{\mathrm{M}}$. Second, the free enthalpy difference $\Delta G_{\mathrm{b}}^{\mathrm{M} \rightarrow \mathrm{M}^{\prime}}$ is calculated by decoupling the bound state (b), i.e., turning off intermolecular interactions of the guest molecule with the environment in a simulation with a host-guest complex in solvent. The latter is divided into three contributions, the difference $\Delta G_{\mathrm{b} \rightarrow \text { tor }}^{\mathrm{M}}$ by turning on translational and orientational restraints (tor) between host and guest in order to guarantee that the guest molecule stays within the host when turning off intermolecular interactions $\Delta G_{\mathrm{tor}}^{\mathrm{M} \rightarrow \mathrm{M}^{\prime}}$ and lastly turning off the initial restraints $\Delta G_{\text {tor }}^{\mathrm{M}^{\prime}}$. The first two free enthalpy differences can be determined through molecular dynamics simulations by gradually turning on restraints and then turning off the interactions. This was done in one continuous simulation resulting into a combined value $\Delta G_{\mathrm{b} \rightarrow \text { tor }}^{\mathrm{M} \rightarrow \mathrm{M}^{\prime}}=\Delta G_{\mathrm{b} \rightarrow \text { tor }}^{\mathrm{M}}+\Delta G_{\mathrm{tor}}^{\mathrm{M} \rightarrow \mathrm{M}^{\prime}}$. The free enthalpy from turning off the restraints from the non-interacting guest molecule can be calculated analytically. According to the thermodynamic cycle $[34,35]$ shown in figure 2 , the summation over the whole cycle is equal to zero thus resulting into

$$
\begin{aligned}
& \Delta G^{\mathrm{DD}}=\Delta G_{\mathrm{u} \rightarrow \mathrm{b}}^{\mathrm{M}}= \\
& -\Delta G_{\text {hyd }}^{\mathrm{M}}-\Delta G_{\mathrm{b} \rightarrow \text { tor }}^{\mathrm{M}}-\Delta G_{\mathrm{tor}}^{\mathrm{M} \rightarrow \mathrm{M}^{\prime}}-\Delta G_{\mathrm{tor}}^{\mathrm{M}^{\prime}} \\
& =-\Delta G_{\text {hyd }}^{\mathrm{M}}-\Delta G_{\mathrm{b} \rightarrow \text { tor }}^{\mathrm{M} \rightarrow \mathrm{M}^{\prime}}-\Delta G_{\text {tor }}^{\mathrm{M}^{\prime}}
\end{aligned}
$$

The procedure of decoupling the bound ligand was adopted from Boresch and Karplus [36]. Six atoms were chosen, three from the host $a, b, c$ and three from the guest molecule $A, B, C$, see Fig. S2 in the supplementary material. Using these atoms a total of six harmonic restraints have been applied, a distance $r_{a A, 0}$, two angles $\theta_{A, 0}, \theta_{B, 0}$, and three dihedral angles $\phi_{A, 0}, \phi_{B, 0}$, $\phi_{C, 0}$. The values for the reference distance and angles have been determined by averaging the distances and angles of host-guest complexes throughout the unbiased simulations. The analytical part for turning off these restraints is then given by

$$
\begin{aligned}
& \Delta G_{\mathrm{tor}}^{\mathrm{M}^{\prime}}= \\
& \quad-R T \ln \left[\frac{8 \pi V^{0}\left(K_{r} K_{\theta_{A}} K_{\theta_{B}} K_{\phi_{A}} K_{\phi_{B}} K_{\phi_{C}}\right)^{\frac{1}{2}}}{r_{a A, 0}^{2} \sin \theta_{A, 0} \sin \theta_{B, 0}(2 \pi R T)^{3}}\right]
\end{aligned}
$$

with force constants $K_{i}$. Depending on the guest molecule, distinct binding configurations may become apparent. In this case the decoupling has to be performed for each of those states $k$. The total binding free en-



Fig. 2 Thermodynamic cycle for calculating the binding free enthalpy of a host-guest system. Hereby M indicates that the guest molecule is possessing full intermolecular (environmental) interaction (full triangle), while M' denotes the guest molecule possessing only intramolecular interactions (framed triangle). Index u stands for an unbound state, $\mathrm{b}$ for the bound state, and tor for translational and orientational restraints.

thalpy is then calculated by a logarithmic mean [37]

$\Delta \bar{G}_{\mathrm{u} \rightarrow \mathrm{b}}^{\mathrm{M}}=-R T \ln \left[\sum_{k} \exp \left(\frac{\Delta G_{\mathrm{u} \rightarrow \mathrm{b}, k}^{\mathrm{M}}}{R T}\right)\right]$.

\subsection{Immobilization of cyclodextrins}

For immobilizing cyclodextrin in a silica-pore, two linker concepts used by Huq et al. [16] and Trofymchuk et al. [19] were utilized for generating the molecule structure for simulation. Since the native cyclodextrin molecules were described by the Amber-compatible q4mdCD force field [38], the linker molecules were parametrized via AmberTools20 [39]. The parametrized linker structures were then appended to the cyclodextrin topology while accounting for additional connectivity parameters which are listed in the supplementary material in table S3. The molecular structures of the linkers are illustrated in figure 3 .

\subsection{Simulated systems}

For calculating the binding free enthalpy by direct counting and via rate constants, long unbiased simulations were conducted in the $N p T$ ensemble with one guest molecule, benzene or $p$-nitrophenol, respectively, and one host molecule, $\beta$-cyclodextrin, solvated in 1000 water molecules. Simulations for determining the binding 
(a)



(b)

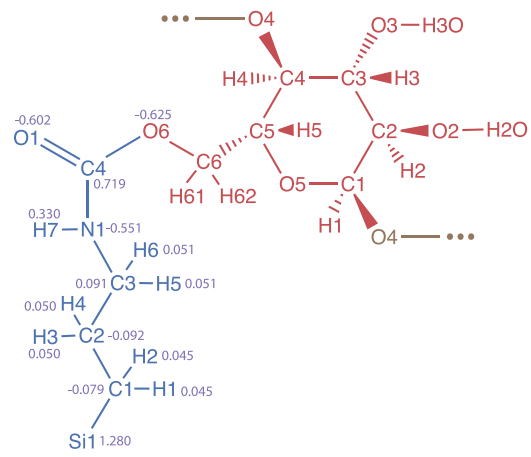

Fig. 3 Atom names and partial atomic charges (purple) of cyclodextrin (red) with a pore surface linker (blue) used by (a) Huq et al. [16] and referred to as L1 in the present work, with a total charge beyond the Si1-atom (not counting the $\mathrm{Na}^{+}$) of $-1.32 e$ and by (b) Trofymchuk et al. [19], referred to as L2 in the present work, with a total charge of $-0.32 e$

free enthalpy by double decoupling were initialized from a configuration of the long unbiased simulations containing a host-guest complex in the bound state. The hydration free enthalpy was calculated from a similar system without a host molecule. The binding free enthalpy was calculated for two temperatures, $298 \mathrm{~K}$ and $350 \mathrm{~K}$ at a constant pressure of 1 bar.

In order to generate and functionalize silica-pores as shown in figure 4, the PoreMS Python package [40] version 0.2.2 [41] was utilized. The systems are composed of a cylindrical pore of $4.05 \mathrm{~nm}$ diameter carved out of a $(6.07,6.14,10.08) \mathrm{nm}(x, y, z) \beta$-cristobalite block. A bulk reservoir with the length of $5 \mathrm{~nm}$ was attached on each side of the pore structure. The internal surface was functionalized with 0.07 and $0.14 \mu_{\mathrm{mol} \mathrm{m}}{ }^{-2}$ $\beta$-cyclodextrin, respectively. For the simulations representing the system by Trofymchuk et al. [19], additional 0.11 and $0.22 \mathrm{\mu mol} \mathrm{m}^{-2}$ of the propylamine group was added to the internal surface, which was experimentally used to immobilize cyclodextrin. This resulted into a total hydroxylation density ( $\mathrm{OH}$-groups on the surface) of $8.56 \mu_{\mathrm{mol} \mathrm{m}}^{-2}$ on the internal surface and
$8.82 \mu \mathrm{mol} \mathrm{m}^{-2}$ on the external surface. Further properties of the pore are listed table 1 .

Table 1 Properties of the cylindrical mesopore model before and after functionalization (func), generated as described by Trofymchuk et al. [19], with surface densities $\left(\mu \mathrm{mol} \mathrm{m}{ }^{-2}\right)$ and pore dimensions $(\mathrm{nm})$.

\begin{tabular}{|c|c|c|}
\hline & Interior & Exterior \\
\hline Silica block $x y z$-dim. & \multirow{2}{*}{\multicolumn{2}{|c|}{$\begin{array}{c}6.07 ; 6.14 ; 10.08 \\
z\end{array}$}} \\
\hline Pore drilling direction & & \\
\hline Pore diameter & \multicolumn{2}{|l|}{4.05} \\
\hline Surface roughness $^{\mathrm{a}}$ & 0.08 & 0.00 \\
\hline Solvent reservoir $z$-dim. & \multicolumn{2}{|r|}{5.00} \\
\hline Simulation box $x y z$-dim. & \multicolumn{2}{|c|}{$6.07 ; 6.14 ; 20.08$} \\
\hline Pore volume & 129.74 & \\
\hline Solvent reservoir volume & \multicolumn{2}{|r|}{$2 \times 186.36$} \\
\hline Surface area & 128.16 & $2 \times 24.39$ \\
\hline \multicolumn{3}{|l|}{ Surface chemistry before func. } \\
\hline Num. of single silanol groups & 553 & 205 \\
\hline Num. of geminal silanol groups & 68 & 27 \\
\hline Num. of siloxane bridges & 0 & 0 \\
\hline Total number of $\mathrm{OH}$ groups & 689 & 259 \\
\hline Overall hydroxylation & 8.93 & 8.82 \\
\hline \multicolumn{3}{|l|}{ Surface chemistry after func. } \\
\hline Num. of $\beta$-cyclodextrin groups ${ }^{b}$ & 11 & 0 \\
\hline$\beta$-cyclodextrin density & 0.14 & 0 \\
\hline Num. of propylamine groups & 17 & 0 \\
\hline Propylamine density & 0.22 & 0 \\
\hline Bonded-phase density & 0.36 & 0.00 \\
\hline Num. of residual $\mathrm{OH}$ groups & 661 & 259 \\
\hline Residual hydroxylation & 8.56 & 8.82 \\
\hline
\end{tabular}

${ }^{a}$ Calculated as the standard deviation of the shortest distances between the central pore axis and the surface Si atoms.

${ }^{\mathrm{b}}$ Pore variants with 5 and $11 \beta$-cyclodextrin groups were prepared in this work.

The topology parameters for the lattice silicon and oxygen atoms and silanol groups are taken from Gulmen and Thompson [42] and are shown in the supplementary material in table $\mathrm{S} 4$. These systems were simulated in the NVT ensemble by achieving the desired density and pressure in a system with a specific concentration of guest molecules, through iteratively filling the simulation box with solute molecules until the difference between the density within the bulk-reservoir of the pore system and a preliminary $N p T$ simulation at a pressure of 1 bar with the same solvent concentration, is less than one percent. Benzene adsorption isotherms were determined for two temperatures, $298 \mathrm{~K}$ and $350 \mathrm{~K}$ and two cyclodextrin surface densities. For $p$-nitrophenol an adsorption isotherm was calculated at $350 \mathrm{~K}$ using the pore with the higher cyclodextrin surface density. 
(a)

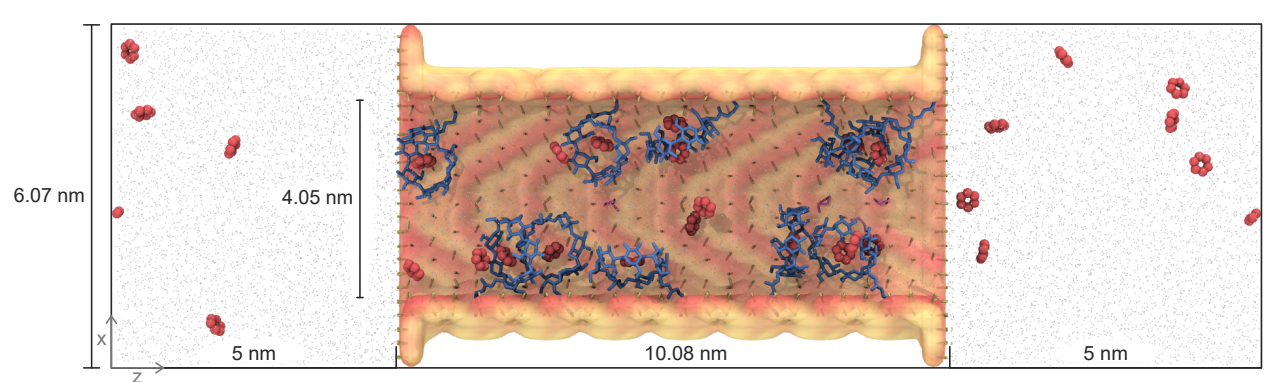

(b)

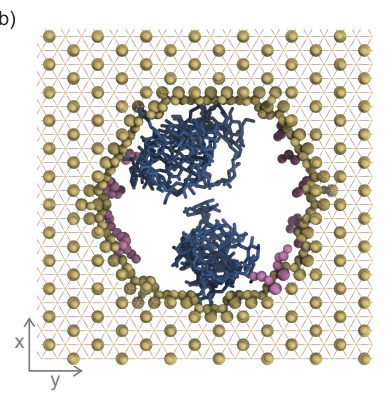

Fig. 4 Pore system functionalized with $\beta$-cyclodextrin using the surface linker used by Trofymchuk et al. [19]. (a) Side view of the simulation box indicating the length of the central silica block and the solvent reservoirs. (b) Front view of the pierced silica block containing the $4 \mathrm{~nm}$ diameter pore. The chemistry of the exterior surface is based on the (111) face of $\beta$-cristobalite silica. Bonded-phase groups are randomly distributed on the silica surface. Ligand densities, residual surface hydroxylation, and further details are specified in Table 1. Colour code: Si, yellow lines; O, red lines; $\beta$-cyclodextrin, blue; propylamine groups, magenta; surface silanol groups, yellow.

\subsection{Simulation parameters}

Simulations were prepared using the open source package PoreSim [43] which generates folder structures and other practical scripts for pore simulations. The simulation suite GROMACS 2016.5 [44, 45] was used for all simulations, while PLUMED $2.5[46,47]$ was utilized to extract specific distances and angles. Based on earlier work [48, 49], the general Amber force field (GAFF) [50] was chosen to describe intra- and intermolecular interactions. This is further backed up by the quality of the GAFF-compatible force field q4md-CD for cyclodextrin simulations $[38,51]$. In order to verify the quality of the force-fields for the solute molecules $p$-nitrophenol and benzene, which were studied in the work of Huq et al. [16] and Trofymchuk et al. [19], validating simulations were carried out based on topologies provided by Mobley et al. [52]. Water was described by the TIP3P model $[53]$.

All MD simulations were performed under periodic boundary conditions. Temperature was controlled using the Nosé-Hoover thermostat $[54,55]$ with a coupling constant of $1 \mathrm{ps}$, while pressure for simulations in the $N p T$ ensemble was controlled by the ParrinelloRahman barostat $[56,57]$ with a coupling constant of $5.0 \mathrm{ps}$ and compressibility of $4.5 \times 10^{-5} \mathrm{bar}^{-1}$. Bond lenghts between heavy atoms and hydrogens were constrained with the LINCS algorithm [58, 59] with an order of 4 . Short-range electrostatic and Lennard-Jones parameters were evaluated up to a cutoff distance of $1.4 \mathrm{~nm}$. Analytical dispersion corrections for energy and pressure were included. Long-range electrostatic interactions were treated with the particle-mesh Ewald algorithm [60, 61].

The long unbiased simulations were run for $10 \mu \mathrm{s}$ with a time-step of 2 fs after a total equilibration time of 3 ns. Decoupling simulations used a total of 25 interme- diate states ( $\lambda$-points) for a slow equidistant deactivation of the intermolecular interactions of the guest molecule with its environment while preserving intramolecular interactions. Each $\lambda$-point was run for $1 \mathrm{~ns}$ with a time-step of $2 \mathrm{fs}$. During the simulation of the pore systems, silicon and oxygen grid atoms were frozen in their position to preserve the original pore shape, this includes the silicon atom of surface groups. For these systems a trajectory length of $1 \mu$ s was generated with a time-step of $1 \mathrm{fs}$ and a total equilibration time of $100 \mathrm{~ns}$.

\subsection{Analysis}

Distances and angles between the reference systems during the long unbiased simulations were extracted using PLUMED. The determination of bound and unbound states, and the calculation of the binding free enthalpy using the direct counting and rate constants method was conducted by in-house Python scripts. For determining the free enthalpy differences from decoupling simulations thermodynamic integration [62] was utilized. The density profiles and adsorption isotherms were calculated using the PoreAna package version 0.2.0 [63], developed during this project in object oriented Python 3 to complement the PoreMS package. Radial density and diffusion profiles within the pore were calculated by dividing the cylindrical shape into radial volume slices

$V_{i}=\pi z\left(r_{i}^{2}-r_{i-1}^{2}\right)$

with pore length $z$ and radius $r_{i}$ of slice $i$. The number density $\rho_{i}$ is then determined by counting the number of molecules $N_{i}$ within each slice during the simulation, resulting into

$\rho_{i}=\frac{N_{i}}{V_{i}}=\frac{N_{i}}{\pi z} \frac{1}{r_{i}^{2}-r_{i-1}^{2}}$. 
Adsorption isotherms describe the amount of solute molecules adsorbed on the surface $N^{\text {pore }}$ as a function of the amount of solute in the bulk phase $N^{\text {bulk }}$ and are therefore, similarly to the density, determined from counting the number of molecules within the pore and within the bulk reservoirs normalized by the number of frames

$$
\begin{aligned}
\langle N\rangle^{\text {pore }} & =\frac{1}{N_{\mathrm{F}}} \sum_{j=1}^{N_{\mathrm{F}}} N_{j}^{\text {pore }}, \\
\langle N\rangle^{\text {bulk }} & =\frac{1}{N_{\mathrm{F}}} \sum_{j=1}^{N_{\mathrm{F}}} N_{j}^{\text {bulk }} .
\end{aligned}
$$

with the number of molecules $N_{j}$ within the pore or bulk phase at frame $j=1, \ldots, N_{\mathrm{F}}$. These values are then converted to surface and volume concentrations respectively based on the volume of the pore system and inner pore surface.

The diffusion coefficient $D_{\|, i}$ parallel to the pore surface was determined from the slope of the mean square displacement $\Delta_{i}(t)$ over an observation time of 4-20 ps within each bin with a tolerance of \pm 1 bins

$D_{\|, i}=\frac{1}{2} \frac{d \Delta_{i}(t)}{d t}$

By weighting the axial diffusion profile $D_{\|, i}$ with the density profile $\rho_{i}$ along the radius $r$, a mean diffusion coefficient $\left\langle D_{\|}\right\rangle$can be calculated

$\left\langle D_{\|}\right\rangle=\frac{\sum \rho_{i} D_{\|, i} A_{i}}{\sum \rho_{i} A_{i}}=\frac{\sum \rho_{i} D_{\|, i}\left(r_{i}^{2}-r_{i-1}^{2}\right)}{\sum \rho_{i}\left(r_{i}^{2}-r_{i-1}^{2}\right)}$

with the cross-sectional area

$A_{i}=\pi\left(r_{i}^{2}-r_{i-1}^{2}\right)$

of bin $i$.

\subsection{Langmuir model}

Considering a simulation set-up as shown in Fig. 4 but with only a single cyclodextrin molecule bound to the inner pore surface and a single guest molecule present in the simulation box, the direct counting method (Eq. 1) would result in the same standard binding free enthalpy as in the bulk phase simulation given that the pore walls do not interact substantially with the guest molecule. Therefore, the ratio of bound to unbound samples in the pore system would be

$$
\left(\frac{N_{\mathrm{b}}}{N_{\mathrm{u}}}\right)_{\text {pore }}=\left(\frac{N_{\mathrm{b}}}{N_{\mathrm{u}}}\right)_{\mathrm{bulk}} \cdot \frac{V_{\mathrm{box}}^{\mathrm{bulk}}}{V_{\mathrm{box}}^{\text {pore }}}
$$

where the sub- or superscript 'pore' refers to the entire accessible volume of the simulation box, containing the pore and the solvent reservoirs. Rearranging to

$N_{\mathrm{b}}^{\text {pore }}=\left(\frac{N_{\mathrm{u}}}{V_{\mathrm{box}}}\right)_{\text {pore }} \cdot\left(\frac{N_{\mathrm{b}}}{N_{\mathrm{u}}}\right)_{\mathrm{bulk}} \cdot V_{\mathrm{box}}^{\mathrm{bulk}}$

and replacing the two rightmost terms by means of Eq. 1 results in

$N_{\mathrm{b}}^{\mathrm{pore}}=\left(\frac{N_{\mathrm{u}}}{V_{\mathrm{box}}}\right)_{\text {pore }} \cdot V^{0} \cdot \exp \left(-\frac{\Delta G^{\mathrm{DC}}}{R T}\right)$.

The first term on the right-hand side can be identified with the bulk concentration of the guest molecule such that the equation has the form of the Henry isotherm. If we assume that each cyclodextrin can only accommodate one guest molecule and relate the amount adsorbed to the inner pore surface we obtain the Langmuir form

$q_{\mathrm{ads}}=q_{\max } \frac{K \cdot C}{1+K \cdot C}$

where $q_{\max }$ denotes the cyclodextrin density inside the pore and

$K=\frac{1}{C^{0}} \exp \left(-\frac{\Delta G}{R T}\right)$

with $C^{0}$ as the concentration of the standard state, i.e. $1 \mathrm{moll}^{-1}$ and $\Delta G$ as the standard binding free enthalpy obtained via double decoupling, direct counting or any other suitable computational or experimental approach. In that way, a Langmuir isotherm can be computed and compared to isotherms obtained from experiments or molecular simulation to assess whether other processes such as binding of multiple guest molecules to one cyclodextrin or cooperative effects of cyclodextrin molecules in close vicinity on the surface are likely to occur.

\section{Results and discussion}

\subsection{Bulk phase simulations}

In order to verify the quality of the force field for the solute molecules $p$-nitrophenol and benzene, validating simulations were carried out based on topologies provided by Mobley et al. [52] resulting in liquid density values in good agreement with experiment, see table 2 . Hydration free enthalpies $\Delta G_{\text {hyd }}$ reported by the Mobley group [64] were reproduced by decoupling simulations of these molecules in water, which in turn are in fair ( $p$-nitrophenol) or good (benzene) agreement with the experimental values. 
Table 2 Liquid densities $\rho\left(\mathrm{kg} \mathrm{m}^{-3}\right)$ for $p$-nitrophenol $(p$-NP) and benzene (BEN) compared with experimental data [65]. Hydration free enthalpy $\Delta G_{\text {hyd }}\left(\mathrm{kJ} \mathrm{mol}^{-1}\right)$ at $298 \mathrm{~K}$ was calculated via simulation ( $\mathrm{sim}$ ) and compared to simulation values reported by the Mobley group [64] (mo) and experimental values (exp) from Cabani et al. [66].

\begin{tabular}{rrrrrr}
\hline & $\rho^{\text {sim }}$ & $\rho^{\text {exp }}$ & $\Delta G_{\text {hyd }}^{\text {sim }}$ & $\Delta G_{\text {hyd }}^{\text {mo }}$ & $\Delta G_{\text {hyd }}^{\text {exp }}$ \\
\hline$p$-NP & $1274.75^{\mathrm{a}}$ & $1283.15^{\mathrm{a}}$ & -35.35 & -35.44 & -44.08 \\
BEN & $861.24^{\mathrm{b}}$ & $873.49^{\mathrm{b}}$ & -3.23 & -3.39 & -3.62 \\
\hline
\end{tabular}

Determined at ${ }^{\mathrm{a}} 387 \mathrm{~K},{ }^{\mathrm{b}} 298 \mathrm{~K}$.

Table 3 shows the binding free enthalpies and rate constants of CD+p-nitrophenol and CD+benzene complexes. For the long unbiased simulations first, a spatial cutoff had to be defined in order to differentiate between bound and unbound states. Since $\beta$-Cyclodextrin has a radius of gyration around $0.6 \mathrm{~nm}$ [67], the spatial cutoff distance was chosen at $0.7 \mathrm{~nm}$ to account for binding on the inner edge of the host molecule. This assumption is further strengthened by figure 5 where the histogram maximum of the center of mass distances between host and guest molecules diminishes at the chosen cutoff and a smaller cluster emerges that indicates the unbound states. The effect of the temporal cut-off between 0 and

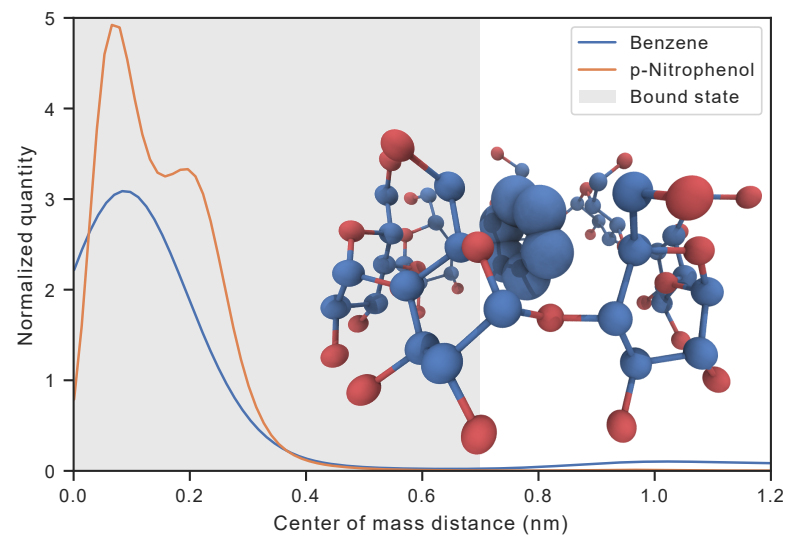

Fig. 5 Histogram of the central distances $\mathbf{C}-\mathbf{C}$ with a marked area of the bound states (grey) of the reference systems for host and guest molecules as illustrated in figure 1 for benzene (blue) and $p$-nitrophenol (orange) at $T=350 \mathrm{~K}$ with the corresponding image showing benzene in the bound state.

$1 \mathrm{~ns}$ on the binding free enthalpy is summarized in table S1 in the supplementary material. The consideration behind the temporal cut-off is that the average residence time of the guest molecule inside the host is usually shorter than an average experimentally determined survival time because in an experiment several shortterm events are likely to be missed. This effect has to be kept in mind when comparing to experimentally determined rates which may differ depending on the tem- poral resolution of the measurement device. For benzene the effect on $\Delta G$ is minor, while for $p$-nitrophenol an effect is only visible between temporal cut-offs of 0 ps and 100 ps. For both molecules the $k_{\mathrm{on}^{-}}$and $k_{\text {off }}-$ rates calculated with 0 ps cut-off are an order of magnitude larger compared to the values calculated with a larger cut-off. In the present work only bound/unbound periods that lasted longer than 1 ns were counted as one bound/unbound event, in agreement with previous works $[32,68]$.

Table 3 Comparison of direct counting (DC), rate constants (RC) and double decoupling (DD) methods for determining the binding free enthalpy $\Delta G\left(\mathrm{~kJ} \mathrm{~mol}^{-1}\right)$ with experimental (Exp) data of $\beta$-cyclodextrin-ligand complexes, with association $k_{\text {on }}$ $\left(10^{8} \mathrm{dm}^{3} \mathrm{~mol}^{-1} \mathrm{~s}^{-1}\right)$ and dissociation $k_{\mathrm{off}}\left(10^{6} \mathrm{~s}^{-1}\right)$ rates at a temporal cut-off of $1 \mathrm{~ns}$ calculated at temperature $T(\mathrm{~K})$. The chosen spatial cut-off for differentiating between bound and unbound states is $0.7 \mathrm{~nm}$ for both guest molecules $p$-nitrophenol $(p-\mathrm{NP})$ and benzene (BEN).

\begin{tabular}{|c|c|c|c|c|c|c|}
\hline \multirow[t]{2}{*}{$T$} & \multicolumn{3}{|c|}{298} & \multicolumn{3}{|c|}{350} \\
\hline & $-\Delta G$ & $k_{\mathrm{on}}$ & $k_{\mathrm{off}}$ & $-\Delta G$ & $k_{\mathrm{on}}$ & $k_{\text {off }}$ \\
\hline \multicolumn{7}{|l|}{$p$-NP } \\
\hline $\mathrm{DC}$ & & & & 24.22 & & \\
\hline $\mathrm{RC}$ & & & & 17.01 & 11.6 & 3.34 \\
\hline $\mathrm{DD}$ & 25.79 & & & 24.07 & & \\
\hline Mean $^{\mathrm{a}}$ & & & & 24.15 & & \\
\hline $\operatorname{Exp}[69,70]$ & $10-17$ & & & & & \\
\hline \multicolumn{7}{|l|}{$\mathrm{BEN}$} \\
\hline $\mathrm{DC}$ & 13.25 & & & 14.11 & & \\
\hline $\mathrm{RC}$ & 13.27 & 5.41 & 2.55 & 13.46 & 8.58 & 8.41 \\
\hline DD & 13.12 & & & 12.15 & & \\
\hline Mean $^{\mathrm{b}}$ & 13.21 & & & 13.24 & & \\
\hline $\operatorname{Exp}[71]$ & $12-13$ & & & & & \\
\hline
\end{tabular}

Mean value from ${ }^{\mathrm{a}} \mathrm{DC}, \mathrm{DD}$, and ${ }^{\mathrm{b}} \mathrm{DC}, \mathrm{RC}, \mathrm{DD}$.

For CD+benzene all three approaches used to calculate the binding free enthalpy yield a consistent value at both temperatures. For CD $+p$-nitrophenol the counting approaches did not provide reliable values at $298 \mathrm{~K}$ due to the rather strong binding and thus low occurrences of unbound instances. The time evolution of bound and unbound instances are provided in the supporting information in figure S1. Therefore only the double decoupling results are reported at this temperature. At $350 \mathrm{~K}$ unbound occurrences are more likely due to the higher temperature, allowing an improved sampling which leads to results for the direct counting approach that is in good agreement with the double decoupling method. Nevertheless, the drop of the binding free enthalpy in the rate constants approach indicates that longer unbound instances are still infrequent. The reason for the larger binding free enthalpy at a higher temperature for benzene in the un- 


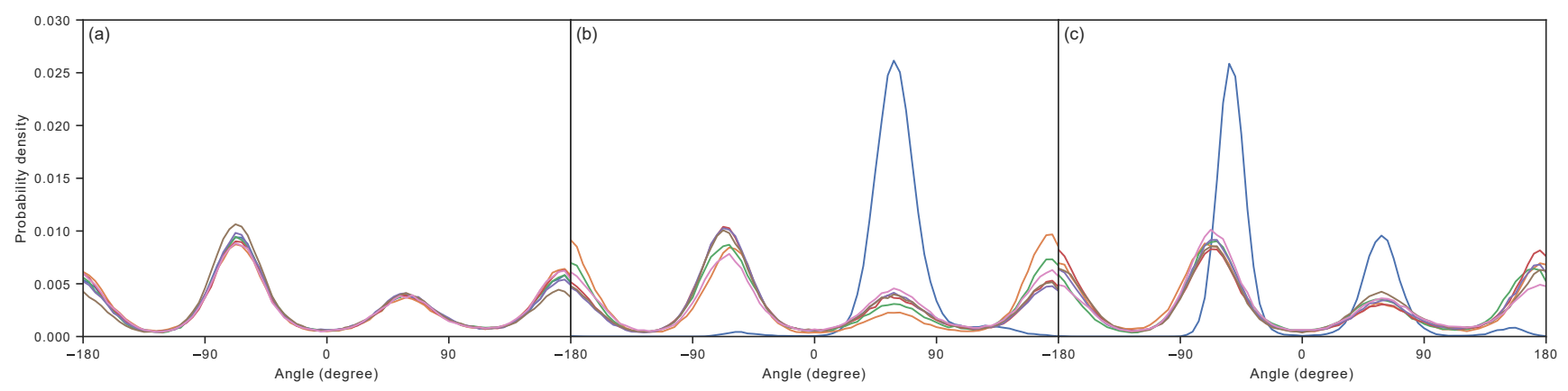

Fig. 6 (a) Distributions of the seven individual dihedral angles H6O-O6-C6-H61 for the native $\beta$-cyclodextrin resulting from an $N p T$ simulation in TIP3P water. The different colours denote the different glucopyranose units. (b) Distribution of six dihedral angles H6O-O6-C6-H61 for $\beta$-cyclodextrin connected with the L1 linker [16]. The blue line represents the distribution of the C5(tail)-O6-C6-H61 dihedral angle. (c) Analogous to (b) but for the L2 linker [19] with the blue line representing the distribution of the C4(tail)-O6-C6-H61 dihedral angle.

biased approaches is due to a larger volumetric correction in equation (1) at $R T_{298} \ln \frac{V_{\text {box }}}{V 0}=7.28 \mathrm{~kJ} \mathrm{~mol}^{-1}$ and, $R T_{350} \ln \frac{V_{\text {box }}}{V^{0}}=8.69 \mathrm{~kJ} \mathrm{~mol}^{-1}$, which is not entirely compensated by the decrease of the ratio of bound and unbound instances $R T_{298} \ln \frac{N_{\mathrm{b}}}{N_{\mathrm{u}}}=5.98 \mathrm{~kJ} \mathrm{~mol}^{-1}$ and, $R T_{350} \ln \frac{N_{\mathrm{b}}}{N_{\mathrm{u}}}=5.41 \mathrm{~kJ} \mathrm{~mol}^{-1}$, as they stay almost similar.

For assessing the properties of the parametrized CD molecule with an attached linker for connecting to the pore surface, $N p T$ simulations in TIP3P water were carried out and the resulting dihedral-angle distributions were analyzed. Figure 6(a) shows that for the native cyclodextrin the distributions are in good agreement with those reported by Gebhardt et al. [51] using the same force field. Additional dihedral-angle distributions for $\alpha$ - and $\gamma$-cyclodextrin are shown in the supplementary material in figure S3. Attaching the linker affects the corresponding dihedral angle describing rotation around the C6-O6 bond, see figure 3. With the L1-variant essentially one rotamer around $60^{\circ}$ is populated (figure 6(b)) while the L2-variant has two rotamers populated (figure 6(c)).

\subsection{Unfunctionalized silica pores}

Figure 7 shows density profiles of water, benzene, and $p$-nitrophenol as well as axial diffusion coefficient profiles in a system containing 60 solute molecules which corresponds to a concentration of $200 \mathrm{mmoll}^{-1}$. Hardly any adsorption for benzene or $p$-nitrophenol is visible, in agreement with experiments $[16,19]$. The number densities converges rapidly towards the density value at the pore center. The slowdown of water diffusion in confinement relative to the bulk phase can be compared with recent experimental data reported by Jani et al. [72]. At a temperature of $300 \mathrm{~K}$ the experimen- tal self-diffusion coefficient of water in the bulk phase, $2.3 \times 10^{-9} \mathrm{~m}^{2} \mathrm{~s}^{-1}$, is reduced to $2.0 \times 10^{-9} \mathrm{~m}^{2} \mathrm{~s}^{-1}$ in SBA-15 with a pore mean pore diameter of $6.6 \mathrm{~nm}$ and to $1.7 \times 10^{-9} \mathrm{~m}^{2} \mathrm{~s}^{-1}$ in MCM-41 with a pore diameter of $3.8 \mathrm{~nm}$, close to the simulated pore diameter of $4 \mathrm{~nm}$. This accounts to change of factor 1.15 for the SBA15 system and 1.35 in the MCM-41 pore. The mean diffusion $\left\langle D_{\|}\right\rangle$of water in the simulated unfunctionalized pore is $3.07 \times 10^{-9} \mathrm{~m}^{2} \mathrm{~s}^{-1}$ at a temperature of $298 \mathrm{~K}$ and $5.63 \times 10^{-9} \mathrm{~m}^{2} \mathrm{~s}^{-1}$ at a higher temperature of $350 \mathrm{~K}$. This yields a factor 1.70 at $298 \mathrm{~K}$ and 1.74 at $350 \mathrm{~K}$ by comparing the pore diffusion to the bulk diffusion of the same system.

\subsection{Cyclodextrin-functionalized silica pores}

By functionalizing the surface, benzene molecules have a significantly higher concentration at the configurational space of the cyclodextrin center of mass, indicating host-guest interaction, see figure 8.

Benzene adsorption isotherms were calculated for a low cyclodextrin surface concentration at $298 \mathrm{~K}$ and a high cyclodextrin concentration at $298 \mathrm{~K}$ and $350 \mathrm{~K}$. For $p$-nitrophenol only the larger surface concentration at $350 \mathrm{~K}$ was considered due to the strong binding affinity that leads to unreliable statistics at the lower temperature. The volumetric and the surface concentration of the guest molecules were assessed. The volumetric concentration in the reservoir was converted from the average number of molecules, which was determined by counting the solute molecule occurrences inside the reservoir normalized by the number of frames. Similarly, a surface specific concentration was determined by counting the appearances of the solute molecules inside the pore. In order to obtain excess adsorption, the adsorption value of the solute molecules within a nonfunctionalized pore has been subtracted. Repeating the 


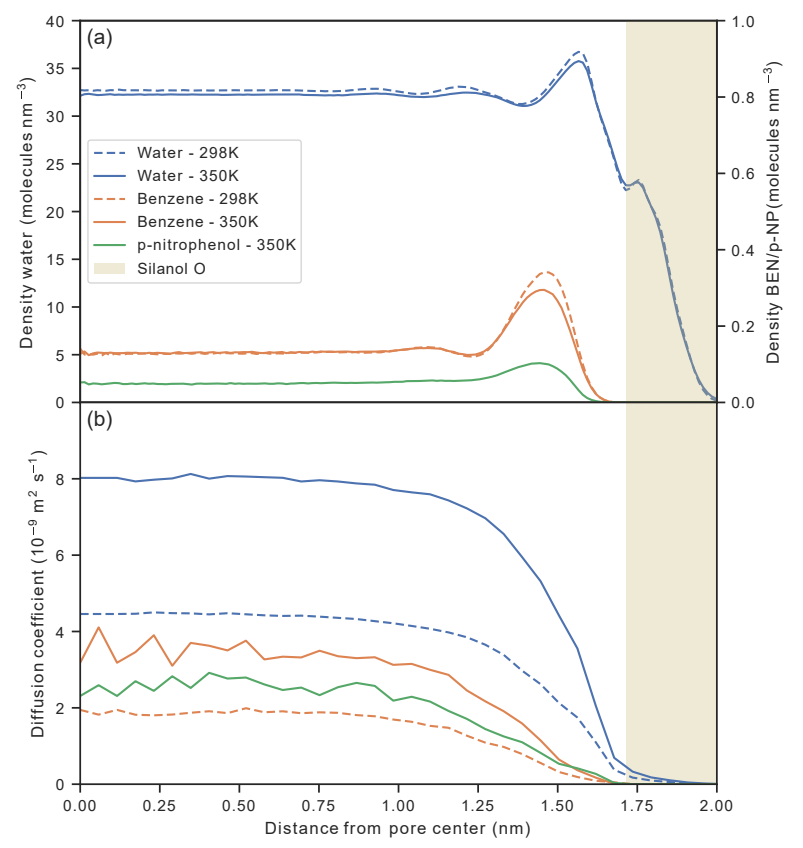

Fig. 7 (a) Density and (b) axial diffusion profiles of water (blue), benzene (BEN) (orange), and p-nitrophenol (p-NP) (green) in a non-functionalized pore at temperatures $298 \mathrm{~K}$ (dashed lines) and $350 \mathrm{~K}$ (solid lines). The shaded area denotes the configurational space of the silanol group oxygen atoms (yellow).

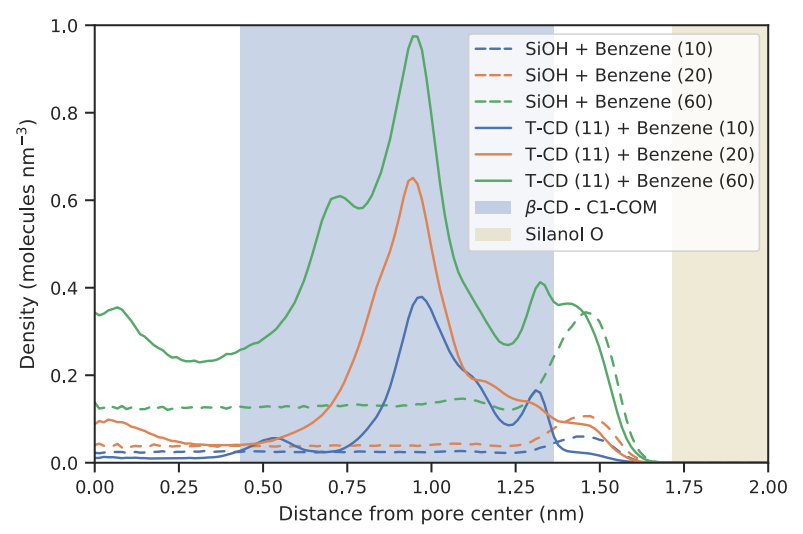

Fig. 8 Radial density profile of the benzene centre of mass in a non-functionalized pore (dashed lines) and in a functionalized pore using the L2-variant cyclodextrin (T-CD) [19] (solid lines) simulated for $1 \mu \mathrm{s}$. Shaded areas denote the configurational space of the cyclodextrin centre of mass (blue) and the silanol groups oxygen atoms (yellow).

pore simulation for different solute concentrations in the system, results into adsorption isotherms shown in figure 9. Further isotherms are provided in figure S4 of the supplementary material

Similar to experimental observation a simple relation between the amount adsorbed and the number of cyclodextrin molecules attached to the surface could only be observed at small concentration, following Lang- muir behavior. For benzene, Trofymchuk et al. [19] reported adsorption isotherms for materials with different amounts of cyclodextrin molecules up to benzene concentrations of $7 \mathrm{mmoll}^{-1}$, i.e., roughly one third of the solubility limit of $23.8 \mathrm{mmol}^{-1}$ at $25^{\circ} \mathrm{C}$ [73]. The corresponding amount adsorbed exceeded the capacity of a 1:1 binding by more than a factor of 10 and the isotherms showed dual-site Langmuir type behavior. In the present work higher benzene concentrations of up to $60 \mathrm{mmoll}^{-1}$ were used to improve the statistical sampling. However, the amount adsorbed did not exceed the 1:1 binding capacity by more than a factor of two. The visual inspection of the trajectories shows that for the lower cyclodextrin density (i.e. five molecules attached to the pore surface) up to three benzene molecules may be associated to one cyclodextrin molecule. For the higher cyclodextrin density some cyclodextrin molecules may also be trapped in the space between the pore surface and the outer surface of the cyclodextrin, thus enhancing the adsorption capacity. Moreover, cyclodextrin molecules in close vicinity may associate and from rather long-living complexes that encapsulate the solute molecules. In addition, a benzene molecule dissociating from one cyclodextrin is very likely associating with the next one close by instead of leaving the pore.

For $p$-nitrophenol experimental adsorption isotherms were reported for very low bulk concentrations below $0.15 \mathrm{mM}$, compared to the aqueous solubility of 115 $\mathrm{mM}$ at $25^{\circ} \mathrm{C}$ [74]. In this regime Langmuir behavior was observed with the Langmuir constant resembling the 1:1 association free enthalpy, as expected [17]. In the simulations, a larger range of bulk-phase concentration has been studied. The binding free enthalpy for the 1:1 complex is overestimated by the force field, leading to rather strong increase in the amount adsorbed at low bulk phase concentration that first follows the Langmuir isotherm but exceeds the 1:1 binding capacity by more than a factor of three due to the association of three nitrophenol molecules with on cyclodextrin molecules as well as the trapping between cyclodextrin and the pore surface.

\section{Conclusion and outlook}

An efficient model building is a prerequisite for computational studies of functionalized mesoporous silica materials. The Python package PoreMS introduced previously $[40,41]$ was complemented by two additional program packages for preparing MD simulations of porous materials with GROMACS, PoreSim [43], and for analyzing the simulation trajectories, PoreAna [63], the latter providing results such as density and diffusion profiles, thereby reducing the overhead for system prepara- 


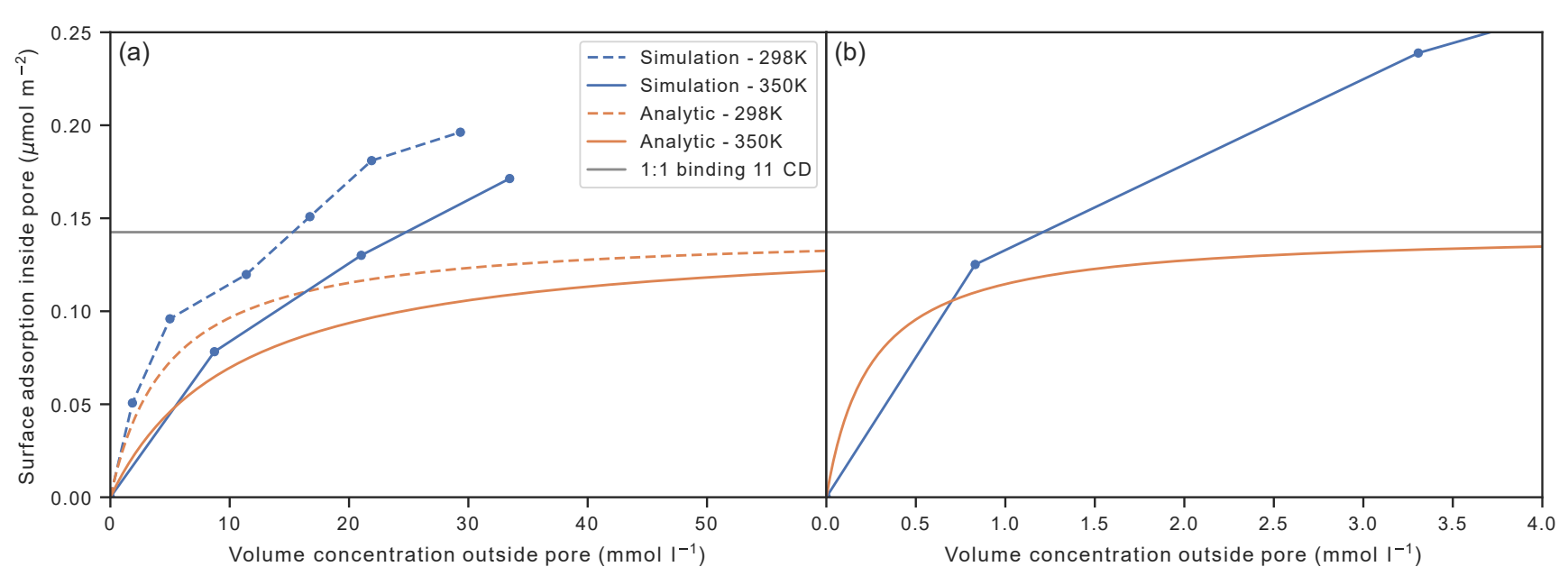

Fig. 9 (a) Excess adsorption isotherms of pore simulations utilizing a Trofymchuk-variant [19] cyclodextrin functionalized surface (blue) compared to the analytical solution shown in equation (16) (orange) at varying amounts of benzene. The binding free enthalpy used to evaluate equation (16) was calculated as the mean value of all utilized methods (DC, RC, DD) at the respective temperature. (b) Like (a) but with a Huq-variant [16] cyclodextrin with p-nitrophenol and the mean free enthalpy value only from the DD and DC approach. Simulation were run for $1 \mu$ s at $298 \mathrm{~K}$ (dashed lines) and $350 \mathrm{~K}$ (solid lines). The grey line represents a hypothetical maximum of 1:1 binding with 11 cyclodextrin molecules.

tion to analysis. The selected case study of adsorption of aromatic molecules in cyclodextrin-functionalized silica mesopores shows that current moderate computational resources allow an atomistically resolved model $(\sim 65000$ atoms $)$ to be propagated to the $\mu$ s time scale. The calculated adsorption isotherms show a more complex behavior than predicted by a simple Langmuir model corresponding to a 1:1 host:guest binding complex. Beside the formation of 1:2 and even 1:3 host:guest complexes also host-host interactions on the surface as well as more unspecific host-guest interactions have an influence on the shape of the isotherm. The information is relevant, for example, for the prediction of band broadening in liquid chromatography [75]. While the aim of the present work was to investigate the feasibility of calculating liquid phase adsorption isotherms by all-atom MD, future work may address the study of multicomponent mixtures, the influence of the solvent or the investigation of chiral stationary phases, e.g by attaching cyclodextrin derivatives.

Acknowledgements This work was financially supported by the Deutsche Forschungsgemeinschaft (DFG, German Research Foundation) - project-ID 358283783 - SFB 1333 and by the Ministry of Science, Research and the Arts of the State of BadenWürttemberg, Germany. Simulation were performed on the supercomputer ForHLR 2 and its successor HoreKa funded by the Ministry of Science, Research and the Arts Baden-Württemberg and by the Federal Ministry of Education and Research.

\section{Conflict of interest}

The authors declare that they have no conflict of interest.

\section{References}

1. B. Tian, S. Hua, Y. Tian, J. Liu, Environ. Sci. Pollut. R. 28(2), 1317 (2021). DOI 10.1007/s11356020-11168-2

2. H. Lorenz, A. Seidel-Morgenstern, Angew. Chem. Int. Ed. 53(5), 1218 (2014). DOI 10.1002/anie.201302823

3. R. Breslow, S.D. Dong, Chem. Rev. 98(5), 1997 (1998). DOI 10.1021/cr970011j

4. S. Hu, J. Li, J. Xiang, J. Pan, S. Luo, J.P. Cheng, J. Am. Chem. Soc. 132(20), 7216 (2010). DOI 10.1021/ja102819g

5. M.P. van der Helm, B. Klemm, R. Eelkema, Nat. Rev. Chem. 3(8), 491 (2019). DOI 10.1038/s41570019-0116-0

6. M. De Rosa, P. La Manna, C. Talotta, A. Soriente, C. Gaeta, P. Neri, Front. Chem. 6, 84 (2018). DOI 10.3389/fchem.2018.00084

7. P. Rai, M. Srivastava, S. Yadav, J. Singh, J. Singh, Catal. Lett. 145(12), 2020 (2015). DOI 10.1007/s10562-015-1588-2

8. A. Molnár, ChemCatChem 13(6), 1424 (2021). DOI $10.1002 /$ cctc. 202001610 
9. C. Gaeta, P. La Manna, M. De Rosa, A. Soriente, C. Talotta, P. Neri, ChemCatChem 13(7), 1638 (2021). DOI 10.1002/cctc.202001570

10. E. Del Valle, Process Biochem. 39(9), 1033 (2004). DOI 10.1016/S0032-9592(03)00258-9

11. B.V.K.J. Schmidt, C. Barner-Kowollik, Angew. Chem. Int. Ed. 56(29), 8350 (2017). DOI 10.1002 /anie. 201612150

12. G. Gattuso, S.A. Nepogodiev, J.F. Stoddart, Chem. Rev. 98(5), 1919 (1998). DOI $10.1021 / \mathrm{cr} 960133 \mathrm{w}$

13. A.R. Khan, P. Forgo, K.J. Stine, V.T. D'Souza, Chem. Rev. 98(5), 1977 (1998). DOI $10.1021 / \mathrm{cr} 970012 \mathrm{~b}$

14. A. Harada, J. Li, M. Kamachi, Nature 364(6437), 516 (1993). DOI 10.1038/364516a0

15. I. Ciucanu, W.A. König, J. Chromatogr. A 685(1), 166 (1994). DOI 10.1016/0021-9673(94)00564-8

16. R. Huq, L. Mercier, P.J. Kooyman, Chem. Mater. 13(12), 4512 (2001). DOI 10.1021/cm010171i

17. A. Bibby, L. Mercier, Green Chem. 5, 15 (2003). DOI 10.1039/B209251B

18. N.V. Roik, L.A. Belyakova, I.M. Trofymchuk, M.O. Dziazko, O.I. Oranska, J. Nanopart. Res. 19(9), 317 (2017). DOI 10.1007/s11051-017-3999-z

19. I. Trofymchuk, N. Roik, L. Belyakova, Nanoscale Res. Lett. 12(1), 307 (2017). DOI 10.1186/s11671017-2072-2

20. R.I. Derrah, D.M. Ruthven, Can. J. Chem. 53(7), 996 (1975). DOI 10.1139/v75-142

21. A. Dąbrowski, Adv. Colloid. Interface Sci. 93(1-3), 135 (2001). DOI 10.1016/S0001-8686(00)00082-8

22. B. Smit, T.L.M. Maesen, Chem. Rev. 108(10), 4125 (2008). DOI 10.1021/cr8002642

23. B.C. Bukowski, F.J. Keil, P.I. Ravikovitch, G. Sastre, R.Q. Snurr, M.O. Coppens, Adsorption 27, 683 (2021). DOI 10.1007/s10450-021-00314-y

24. R.K. Lindsey, J.L. Rafferty, B.L. Eggimann, J.I. Siepmann, M.R. Schure, J. Chromatogr. A 1287, 60 (2013). DOI 10.1016/j.chroma.2013.02.040

25. S.M. Melnikov, A. Höltzel, A. Seidel-Morgenstern, U. Tallarek, Angew. Chem. Int. Ed. 51(25), 6251 (2012). DOI 10.1002/anie.201201096

26. J. Rybka, A. Höltzel, U. Tallarek, J. Phys. Chem. C 121(33), 17907 (2017). DOI 10.1021/acs.jpcc.7b04746

27. K. El Hage, R.J. Bemish, M. Meuwly, Phys. Chem. Chem. Phys. 20(27), 18610 (2018). DOI 10.1039/C8CP02899K

28. J.E. Basconi, G. Carta, M.R. Shirts, AIChE J. 63, 4564 (2017). DOI 10.1002/aic.15798

29. S. Jakobtorweihen, J. Heuer, T. Waluga, J. Chromatogr. A 1620, 460940 (2020). DOI 10.1016/j.chroma.2020.460940

30. I. Nezbeda, J. Škvára, Mol. Simul. 47, 846 (2020). DOI 10.1080/08927022.2020.1828584

31. F. Gritti, Anal. Chem. 93, 5653 (2021). DOI 10.1021/acs.analchem.0c05078

32. J. Baz, J. Gebhardt, H. Kraus, D. Markthaler, N. Hansen, Chem. Ing. Tech. 90(11), 1864 (2018). DOI $10.1002 /$ cite. 201800050

33. M. Gilson, J. Given, B. Bush, J. McCammon, Biophys. J. 72(3), 1047 (1997). DOI 10.1016/S00063495(97)78756-3

34. W.F. van Gunsteren, H.J.C. Berendsen, J. Comput. Aid. Mol. Des. 1(2), 171 (1987). DOI 10.1007/BF01676960

35. M.L. Mugnai, R. Elber, J. Chem. Theory Comput. 8(9), 3022 (2012). DOI 10.1021/ct3003817

36. S. Boresch, F. Tettinger, M. Leitgeb, M. Karplus, J. Phys. Chem. B 107(35), 9535 (2003). DOI 10.1021/jp0217839

37. D.L. Mobley, J.D. Chodera, K.A. Dill, J. Chem. Phys. 125(8), 084902 (2006). DOI $10.1063 / 1.2221683$

38. C. Cézard, X. Trivelli, F. Aubry, F. Djedaïni-Pilard, F.Y. Dupradeau, Phys. Chem. Chem. Phys. 13(33), 15103 (2011). DOI 10.1039/c1cp20854c

39. D.A. Case, K. Belfon, I.Y. Ben-Shalom, S.R. Brozell, D.S. Cerutti, T.E. Cheatham, III, V.W.D. Cruzeiro, T.A. Darden, R.E. Duke, G. Giambasu, M.K. Gilson, H. Gohlke, A.W. Goetz, R. Harris, S. Izadi, S.A. Izmailov, K. Kasavajhala, A. Kovalenko, R. Krasny, T. Kurtzman, T.S. Lee, S. LeGrand, P. Li, C. Lin, J. Liu, T. Luchko, R. Luo, V. Man, K.M. Merz, Y. Miao, O. Mikhailovskii, G. Monard, H. Nguyen, A. Onufriev, F. Pan, S. Pantano, R. Qi, D.R. Roe, A. Roitberg, C. Sagui, S. Schott-Verdugo, J. Shen, C.L. Simmerling, N.R. Skrynnikov, J. Smith, J. Swails, R.C. Walker, J. Wang, L. Wilson, R.M. Wolf, X. Wu, Y. Xiong, Y. Xue, D.M. York, P.A. Kollman, AMBER 2020 (2020)

40. H. Kraus, J. Rybka, A. Höltzel, N. Trebel, U. Tallarek, N. Hansen, Mol. Simul. 47(4), 306 (2021). DOI 10.1080/08927022.2020.1871478

41. H. Kraus, N. Hansen. Porems: 0.2 .2 (2021). DOI 10.5281/zenodo.4738036

42. T.S. Gulmen, W.H. Thompson, Langmuir 22(26), 10919 (2006). DOI 10.1021/la062285k

43. H. Kraus. Poresim: 0.1 .0 (2021). DOI 10.5281 /zenodo.4588861

44. M.J. Abraham, T. Murtola, R. Schulz, S. Páll, J.C. Smith, B. Hess, E. Lindahl, SoftwareX 1-2, 19 (2015). DOI 10.1016/j.softx.2015.06.001 
45. B. Hess, C. Kutzner, D. van der Spoel, E. Lindahl, J. Chem. Theory. Comput. 4(3), 435 (2008). DOI $10.1021 / \operatorname{ct} 700301 q$

46. M. Bonomi, D. Branduardi, G. Bussi, C. Camilloni, D. Provasi, P. Raiteri, D. Donadio, F. Marinelli, F. Pietrucci, R.A. Broglia, M. Parrinello, Comput. Phys. Commun. 180(10), 1961 (2009). DOI 10.1016/j.cpc.2009.05.011

47. G.A. Tribello, M. Bonomi, D. Branduardi, C. Camilloni, G. Bussi, Comput. Phys. Commun. 185(2), 604 (2014). DOI 10.1016/j.cpc.2013.09.018

48. F. Ziegler, J. Teske, I. Elser, M. Dyballa, W. Frey, H. Kraus, N. Hansen, J. Rybka, U. Tallarek, M.R. Buchmeiser, J. Am. Chem. Soc. 141(48), 19014 (2019). DOI 10.1021/jacs.9b08776

49. F. Ziegler, H. Kraus, M.J. Benedikter, D. Wang, J.R. Bruckner, M. Nowakowski, K. Weißer, H. Solodenko, G. Schmitz, M. Bauer, N. Hansen, M.R. Buchmeiser, ACS Catal. 11, 11570 (2021). DOI 10.1021/acscatal.1c03057

50. J. Wang, R.M. Wolf, J.W. Caldwell, P.A. Kollman, D.A. Case, J. Comput. Chem. 25(9), 1157 (2004). DOI 10.1002/jcc.20035

51. J. Gebhardt, C. Kleist, S. Jakobtorweihen, N. Hansen, J. Phys. Chem. B 122(5), 1608 (2018). DOI 10.1021/acs.jpcb.7b11808

52. D.L. Mobley, M. Shirts, N. Lim, J. Chodera, K. Beauchamp, Lee-Ping. MobleyLab/FreeSolv: Version 0.52 (2018). DOI 10.5281/zenodo.1161245

53. W.L. Jorgensen, J. Chandrasekhar, J.D. Madura, R.W. Impey, M.L. Klein, J. Chem. Phys. 79(2), 926 (1983). DOI 10.1063/1.445869

54. S. Nosé, Mol. Phys. 52(2), 255 (1984). DOI 10.1080/00268978400101201

55. W.G. Hoover, Phys. Rev. A 31(3), 1695-(1985). DOI 10.1103/physreva.31.1695

56. M. Parrinello, A. Rahman, Phys. Rev. Lett. 45(14), 1196 (1980). DOI 10.1103/physrevlett.45.1196

57. M. Parrinello, A. Rahman, J. Appl. Phys. 52(12), 7182 (1981). DOI 10.1063/1.328693

58. B. Hess, H. Bekker, H.J.C. Berendsen, J.G.E.M. Fraaije, J. Comp. Chem. 18(12), 1463 (1997). DOI 10.1002/(sici)1096-987x(199709)18:12¡1463::aidjcc4¿3.0.co;2-h

59. B. Hess, J. Chem. Theory Comput. 4(1), 116 (2008). DOI 10.1021/ct700200b

60. T. Darden, D. York, L. Pedersen, J. Chem. Phys. 98(12), 10089 (1993). DOI 10.1063/1.464397

61. U. Essmann, L. Perera, M.L. Berkowitz, T. Darden, H. Lee, L.G. Pedersen, J. Chem. Phys. 103(19), 8577 (1995). DOI 10.1063/1.470117

62. J.G. Kirkwood, J. Chem. Phys. 3(5), 300 (1935). DOI $10.1063 / 1.1749657$
63. H. Kraus, N. Hansen. Poreana: 0.2 .0 (2021). DOI 10.5281/zenodo. 4738182

64. G. Duarte Ramos Matos, D.Y. Kyu, H.H. Loeffler, J.D. Chodera, M.R. Shirts, D.L. Mobley, J. Chem. Eng. Data 62(5), 1559 (2017). DOI 10.1021/acs.jced.7b00104

65. Dortmund data bank. www.ddbst.com (2014)

66. S. Cabani, P. Gianni, V. Mollica, L. Lepori, J. Solution Chem. 10(8), 563 (1981). DOI 10.1007/BF00646936

67. S.K.S. Al-Burtomani, F.O. Suliman, RSC Adv. 7(16), 9888 (2017). DOI 10.1039/C6RA28638K

68. Z. Tang, C.A. Chang, J. Chem. Theory Comput. 14(1), 303 (2018). DOI 10.1021/acs.jctc.7b00899

69. M.V. Rekharsky, Y. Inoue, Chem. Rev. 98(5), 1875 (1998). DOI 10.1021/cr970015o

70. F. Cramer, W. Saenger, H.C. Spatz, J. Am. Chem. Soc. 89(1), 14 (1967). DOI 10.1021/ja00977a003

71. W. Chen, C.E. Chang, M.K. Gilson, Biophys. J. 87(5), 3035 (2004). DOI 10.1529/biophysj.104.049494

72. A. Jani, M. Busch, J.B. Mietner, J. Ollivier, M. Appel, B. Frick, J.M. Zanotti, A. Ghoufi, P. Huber, M. Fröba, D. Morineau, J. Chem. Phys. 154(9), 094505 (2021). DOI 10.1063/5.0040705

73. F.P. Schwarz, Anal. Chem. 52(1), 10 (1980). DOI 10.1021/ac50051a004

74. C. Achard, M. Jaoui, M. Schwing, M. Rogalski, J. Chem. Eng. Data 41(3), 504 (1996). DOI 10.1021/je950202o

75. B.L. Karger, L.R. Snyder, C. Horvath, An introduction to separation science (John Wiley \& Sons, New York, 1973) 